\title{
Locally convex topologies induced by fuzzy norms
}

\author{
A. K. Katsaras \\ Department of Mathematics, University of Ioannina, 45110 Ioannina, GREECE \\ Email: akatsar@uoi.gr
}

\begin{abstract}
It is shown that a Hausdorff topological vector space $E$ is fuzzy normable iff its topology is a metrizable locally convex topology. Subspaces, product and quotient spaces of fuzzy normed spaces are investigated. Also the notion of the tensor product of two fuzzy normed norms is introduced and it is proved that the induced locally convex topology coincides with the projective tensor product topology.
\end{abstract}

Keywords: Fuzzy sets, fuzzy norms, locally convex topologies

\section{Introduction}

A notion of a fuzzy norm, on a real or convex linear space, was for the first time introduced by the author in [12]. Several other authors gave some other definitions of a fuzzy norm. In this paper we use a definition of a fuzzy norm which is analogous, but not exactly the same, with the one used by T. Bag and S. K. Samanta in [2]. In our definition, a fuzzy seminorm, on a vector space $E$, is a map $N$ from $E$ to the family $\mathcal{D}\left(R^{+}\right)$of all fuzzy subsets $\xi$ of $R^{+}$which are increasing, left continuous and such that $\xi(0)=0$ and $\lim _{t \rightarrow \infty} \xi(t)=1$. Each fuzzy seminorm $N$ on $E$ induces a pseudometrizable locally convex topology $\tau_{N}$ on $E$. This topology is Hausdorff iff $N$ is a fuzzy norm. Several properties of the topology $\tau_{N}$ are investigated. We prove that a Hausdorff topological vector space is fuzzy normable iff it is metrizable and locally convex. We show that every continuous fuzzy seminorm on a subspace of a locally convex space $E$ has a continuous extension to all of $E$. For a complete fuzzy normed space $E$ we give a fixed point theorem which is analogous to the one that holds for contraction mappings on complete metric spaces. We also show that a fuzzy normed space $(E, N)$ is complete iff, for each sequence $\left(x_{n}\right)$ of elements of $E$, for which the supremum $\bigvee_{n} \bigoplus_{k=1}^{n} N\left(x_{n}\right)$ exists in $\mathcal{D}\left(R^{+}\right)$, the series $\sum_{n=1}^{\infty} x_{n}$ converges in $E$. This result is analogous to the one that characterizes the Banach spaces. For a fuzzy normed space $(E, N)$ we give a necessary and sufficient condition for a linear functional on $(E, N)$ or a linear map from $(E, N)$ to a locally convex space $F$, to be continuous. We also give the Hahn Banach theorem for the continuous extensions of continuous linear functionals. Next we study subspaces, product and quotient spaces of fuzzy normed spaces. For a sequence $\left(E_{n}, N_{n}\right)$ of fuzzy seminormed spaces, we define a fuzzy seminorm $N$ on the cartesian product $\prod_{n=1}^{\infty} E_{n}$ for which the corresponding locally convex topology is the product topology. Finally, for $\left(E, N_{1}\right)$ and $\left(F, N_{2}\right)$ fuzzy seminormed spaces, we define the tensor product fuzzy seminorm $N=N_{1} \otimes N_{2}$ on the tensor product $E \otimes F$ and show that $\tau_{N}$ is the projective tensor product of the topologies $\tau_{N_{1}}$ and $\tau_{N 2}$.

\section{Preliminaries}

Let $\mathcal{D}\left(R^{+}\right)$denote the family of all fuzzy subsets $\xi$ of $R^{+}=[0, \infty)$ which are increasing, left continuous and such that $\xi(0)=0$ and $\lim _{t \rightarrow \infty} \xi(t)=1$. For a non-negative real number $r$, we denote by $\tilde{r}$ the element of $\mathcal{D}\left(R^{+}\right)$defined by $\tilde{r}(t)=0$, for $t \leq r$, and $\tilde{r}(t)=1$ when $t>r$. For $\xi \in \mathcal{D}\left(R^{+}\right)$and $r \geq 0, r \xi$ is defined by $r \xi=\tilde{0}$, when $r=0$, and $(r \xi)(t)=\xi\left(r^{-1} t\right)$ if $r>0$. We make $\mathcal{D}\left(R^{+}\right)$into a directed set by defining $\xi \eta$ iff $\xi(t) \geq \eta(t)$ for all $t \geq 0$. For 
$\xi, \eta \in \mathcal{D}\left(R^{+}\right)$, the elements $\xi \oplus \eta$ and $\xi \odot \eta$ are defined by :

$$
\begin{gathered}
\xi \oplus \eta(t)=\sup \left\{\xi\left(t_{1} \wedge \eta\left(t_{2}\right): t=t_{1}+t_{2}\right\},\right. \\
\xi \odot \eta(t)= \begin{cases}0 & \text { if } \mathrm{t}=0, \\
\sup \{\xi(s) \wedge \eta(t / s): s>0\} & \text { if } t>0 .\end{cases}
\end{gathered}
$$

For $\xi \in D\left(R^{+}\right)$and $p>0$ we define $\xi^{p}$ by $\xi^{p}(t)=\xi\left(t^{1 / p}\right)$. Clearly $\xi^{p} \in D\left(R^{+}\right)$. We get easily the following

Lemma $2.1 \quad$ 1. $\left(\xi_{1} \odot \xi_{2}\right)^{p}=\xi_{1}^{p} \odot \xi_{2}^{p}$.

2. $\xi^{p_{1}+p_{2}}=\xi^{p_{1}} \odot \xi^{p_{2}}$.

3. $\left(\xi^{p_{1}}\right)^{p_{2}}=\xi^{p_{1} p_{2}}$.

For $\xi_{1}, \ldots, \xi_{n}$, we define inductively

$$
\oplus_{k=1}^{n} \xi_{k}=\left[\oplus_{k=1}^{n-1} \xi_{k}\right] \oplus \xi_{n} .
$$

We get easily that

$$
\oplus_{k=1}^{n} \xi_{k}(t)=\sup \left\{\bigwedge_{k=1}^{n} \xi_{k}\left(t_{k}\right): t=\sum_{k=1}^{n} t_{k}\right\} .
$$

Let $\mathcal{A}$ be a non-empty subset of $\mathcal{D}\left(R^{+}\right)$. An element $\eta$ of $\mathcal{D}\left(R^{+}\right.$is said to be an upper bound (resp. a lower bound) of $\mathcal{A}$ if $\xi \preceq \eta$ (resp. $\eta \preceq \xi$ ) for all $\xi \in \mathcal{A}$. Since $\tilde{0} \preceq \xi$ for all $\xi, \mathcal{A}$ is always bounded from below. If $\xi_{o}$ is defined by

$$
\xi_{o}(t)=\sup \{\xi(t): \xi \in \mathcal{A}\}
$$

then $\xi_{o}$ is the greatest lower bound of $\mathcal{A}$ and it is denoted by $\inf \mathcal{A}$ or by $\wedge \mathcal{A}$.

Lemma 2.2 A non-empty subset $\mathcal{A}$ of $\mathcal{D}\left(R^{+}\right)$is bounded from above iff

$$
\lim _{t \rightarrow \infty} \inf _{\xi \in \mathcal{A}} \xi(t)=1 .
$$

If $\mathcal{A}$ is such a set and if

$$
\eta_{o}(t)= \begin{cases}0 & \text { for } t=0, \\ \sup _{0<s<t} \inf _{\xi \in \mathcal{A}} \xi(s), & \text { if } t>0,\end{cases}
$$

then $\eta_{o}$ is the smallest upper bound of $\mathcal{A}$

Proof. If $\mathcal{A}$ has an upper bound $\eta$, then $\inf _{\xi \in \mathcal{A}} \xi(t) \geq \eta(t)$ and hence

$$
\lim _{t \rightarrow \infty} \inf _{\xi \in \mathcal{A}} \xi(t)=1 .
$$

Conversely, assume that the condition is satisfied and define $\eta_{o}$ as in the Lemma. Then $\eta_{o}$ is clearly increasing and, using the condition, $\lim _{t \rightarrow \infty} \eta_{o}(t)=1$. Also $\eta_{o}$ is left continuous. Indeed, suppose that $\eta_{o}(t)>\alpha>0$. There exists $0<s<t$ such that $\inf _{\xi \in \mathcal{A}} \xi(s)>\alpha$. If now $s<t_{1}<t$, then $\eta_{o}\left(t_{1}\right)>\alpha$, which proves that $\eta_{o}$ is left continuous. Clearly $\eta_{o}$ is an upper bound for $\mathcal{A}$. Given any upper bound $\eta$ and $\eta(t)>\alpha$, there exists $0<s<t$ with $\eta(s)>\alpha$. Now $\eta_{o}(t) \geq \inf _{\xi \in \mathcal{A}} \xi(s) \geq \eta(s)>\alpha$, which proves that $\eta_{o}(t) \geq \eta(t)$, for all $t$, and hence $\eta_{o} \preceq \eta$. Therefore $\eta_{o}$ is the smallest upper bound for $\mathcal{A}$.

For a non-empty bounded subset $\mathcal{A}$ of $\mathcal{D}\left(R^{+}\right)$, we will denote by $\sup \mathcal{A}$ or by $\bigvee \mathcal{A}$ the least upper bound of $\mathcal{A}$. We omit the proof of the following easily established

Lemma 2.3 1. For $c \geq 0$ and $\xi, \eta, \xi_{k} \in \mathcal{D}\left(R^{+}\right)$we have

$$
c \cdot \oplus_{k=1}^{n} \xi_{k}=\oplus_{k=1}^{n} c \xi_{k} \quad \text { and } \quad c \cdot(\xi \odot \eta)=(c \xi) \odot \eta=\xi \odot(c \eta) .
$$

2. Fort $c \geq 0$ and $p>0$, we have $(c \xi)^{p}=c^{p} \xi^{p}$.

3. For a family $\left\{\xi_{i}: i \in I\right\}$ of elements of $\mathcal{D}\left(R^{+}\right), c \geq 0$ and $\xi \in \mathcal{D}\left(R^{+}\right)$, we have

$$
c \cdot \bigwedge_{i \in I} \xi_{i}=\bigwedge_{i \in I} c \xi_{i}, \quad \xi \oplus \bigwedge_{i \in I} \xi_{i}=\bigwedge \xi \oplus \xi_{i} .
$$

4. If $\left\{\xi_{i}: i \in I\right\}$ is a bounded family of elements of $\mathcal{D}\left(R^{+}\right), c \geq 0$ and $\xi \in \mathcal{D}\left(R^{+}\right)$, then

$$
\xi \oplus \bigvee_{i} \xi_{i}=\bigvee_{i} \xi \oplus \xi_{i}, \quad c \cdot \bigvee_{i \in I} \xi_{i}=\bigvee_{i \in I} c \xi_{i}
$$




\section{Fuzzy seminorms, fuzzy norms}

A fuzzy seminorm, on a vector space $E$ over $K, K=R$ or $C$, is a map $N: E \rightarrow \mathcal{D}\left(R^{+}\right)$such that :

1. $(F N 1): N(0)=\tilde{0}$.

2. $(F N 2): N(x+y) \preceq N(x) \oplus N(y)$.

3. $(F N 3): N(c x)(t)=N(x)\left(t|c|^{-1}\right)$ for each non-zero scalar $c$.

We will denote $N(x)(t)$ by $N(x, t)$.

Lemma 3.1 Let $N$ be a fuzzy seminorm on $E, t>0$ and $)<\alpha<1$. Then then set

$$
W_{t, \alpha}=\{x: N(x, t)>\alpha\}
$$

is absolutely convex and absorbing. Moreover $W_{t, \alpha}=t W_{1, \alpha}$.

Proof Clearly $W_{t, \alpha}=t W_{1, \alpha}$. So it suffices to prove that $W=W_{1, \alpha}$ is absolutely convex and absorbing. If $x \in W$ and $|c| \leq 1$, then $c x=0 \in W$, if $c=0$, while for $c \neq 0$, we have $N(c x, 1)=N\left(|c|^{1}\right) \geq N(x, 1)>\alpha$ which implies that $c x \in W$. Also $W$ is convex. Indeed, let $x, y \in W, 0<t<1, z=t x+(1-t) y$. Then

$$
N(z, 1) \geq N(t x, t) \wedge N((1-t) y, 1-t)=N(x, 1) \wedge N(y, 1)>\alpha
$$

and so $z \in W$. Finally, given $x \in E$, there exists $s>0$ such that $N(x, s)>\alpha$ and so $x \in s W$, which completes the proof.

We get easily the following

Lemma 3.2 Let $t_{i}>0,0<\alpha_{i}<1, i=1,2, t=\min \left\{t_{1}, t_{2}\right\}, \alpha=\max \left\{\alpha_{1}, \alpha_{2}\right\}$. Then $W_{t, \alpha} \subset W_{t_{1}, \alpha_{1}} \cap W_{t_{2}, \alpha_{2}}$.

In view of lemmas 2.2 and 2.3, the family of all $W_{t, \alpha}, t>0,0<\alpha<1$, is a base at zero for a locally convex topology $\tau_{N}$ on $E$. We will denote by $q_{\alpha}=q_{\alpha, N}$ the Minkowski functional of the set $W_{1, \alpha}$, i.e

$$
q_{\alpha}(x)=\inf \{t>0: N(x, t)>\alpha\} .
$$

For $t>0$, the Minkowski functional of $W_{t, \alpha}$ coincides with $t^{-1} q_{\alpha}$. Hence $\tau_{N}$ is the topology generated by the seminorms $q_{\alpha}, 0<\alpha<1$.

Lemma 3.3 For $0<\alpha<1$, we have $q_{\alpha}=\inf _{\beta>\alpha} q_{\beta}$.

Proof. It is clear that $q_{\alpha} \leq q_{\beta}$ when $\alpha<\beta$. On the other hand, let $q_{\alpha}(x)<t<1$. There exists $0<s<t$ such that $x \in s W_{1, \alpha}$, i.e. $N(x, s)>\alpha$. If $N(x, s)>\beta>\alpha$, then $q_{\beta}(x) \leq s<t$. The lemma clearly follows.

If $0<\alpha_{1}<\ldots<\alpha_{n} \uparrow 1$, then, for each $0<\alpha<1$, there exists an $n$ with $\alpha<\alpha_{n}$ and so $q_{\alpha} \leq q_{\alpha_{n}}$. Hence $\tau_{N}$ is generated by a countable family of seminorms and therefore it is pseudometrizable.

Lemma $3.4 \tau_{N}$ is metrizable iff $N$ is a fuzzy norm.

Proof. Suppose that $\tau_{N}$ is metrizable and hence Hausdorff. Given a non-zero element $x$ of $E$, there exists $t>0$ and $0<\alpha<1$ such that $x \notin W_{t, \alpha}$, i.e $N(x, t) \leq \alpha$ which implies that $N(x) \neq \tilde{0}$ and so $N$ is fuzzy norm. The converse also follows easily.

Lemma 3.5 Let $N$ be a fuzzy seminorm on $E, t>0,0<\alpha<1$. Then:

1. $N(x, t)>\alpha \Leftrightarrow q_{\alpha}(t)<t$.

2. $N(x, t)=\sup \left\{\beta: q_{\beta}(x)<t\right\}$.

3. $N\left(x, t^{+}\right) \geq \alpha \Leftrightarrow \sup _{\beta<\alpha} q_{\beta}(x) \leq t$.

4. $\{x: N(x, t) \geq \alpha\}=\bigcap_{0<\beta<\alpha}\left\{x: q_{\beta}(x)<t\right\}$.

5. If $q_{\alpha}(x)=t$, then $N(x, t)=\alpha$ iff $q_{\beta}(x)<t$ for each $0<\beta<\alpha$.

6. If $N(x, t)=\alpha$, then $q_{\alpha}(x)=t$ iff $N(x, s)>\alpha$ for all $s>t$. 
Proof. (1) If $q_{\alpha}(x)<t$, then $q_{\alpha}\left(t^{-1} x\right)<1$ and so $t^{-1} x \in W_{1, \alpha}$, i.e. $\left.N(x, t)=N\left(t^{-1} x\right), 1\right)>\alpha$. Conversely assume that $N(x, t)>\alpha$. Choose $<s<t$ with $N(x, s)>\alpha$. Then $q_{\alpha}(x) \leq s<t$.

(2) It follows easily from (1).

(3)Suppose that $N\left(x, t^{+}\right) \geq \alpha$ and let $\beta<\alpha$. For $s>t$, we have $N(x, s) \geq \alpha>\beta$ and so $q_{\beta}(x)<s$. This, being true for each $s>t$, implies that $q_{\beta}(x) \leq t$, for all $0<\beta<\alpha$ and thus $d=\sup _{\beta<\alpha} q_{\beta}(x) \leq t$. Conversely, assume that $d \leq t$ and let $s>t$. Then, for $\beta<\alpha$, we have $q_{\beta}(x)<s$ and therefore $N(x, s)>\beta$. Since this holds for each $\beta>\alpha$, we get that $N(x, s) \geq \alpha$. It follows that $N\left(x, t^{+}\right) \geq \alpha$.

(4) and (6) follow from (1) while (5) follows from (1) and (4). For $N_{1}, N_{2}$ fuzzy seminorms on $E$, the following are equivalent.:

1. $N_{1}(x) \preceq N_{2}(x)$ for all $x \in E$.

2. $q_{\alpha, N_{1}} \leq q_{\alpha, N_{2}}$ for all $0<\alpha<1$.

Corollary 3.6 For $N$ a fuzzy seminorm on $E, t>0$ and $0<\alpha<1$, the set $W_{t, \alpha}$ is open.

Example 3.7 Let $E$ be a non-trivial normed space. For $x \in E$, define $N(x)$ on $[0, \infty)$ by

$$
N(x, t)= \begin{cases}1 & \text { if } t>\|x\|, \\ 0 & \text { if } t \leq\|x\| .\end{cases}
$$

Then $N$ is a fuzzy norm on $E$ and $q_{\alpha, N}=\|\cdot\|$ for all $0<\alpha<1$. In this case we have

$$
A=\{x: N(x, t)<1\}=\{x:\|x\| \geq t\}
$$

and so $A$ is not open.

Theorem 3.8 Let $\left\{p_{\alpha}: 0<\alpha<1\right\}$ be an increasing family of seminorms on a vector space $E$ over $K$ and, for $x \in E$, define

$$
N(x): R^{+} \rightarrow[0,1], \quad N(x, t)=\sup \left\{\alpha: p_{\alpha}(x)<t\right\}
$$

(the supremum over the empty family is taken to be zero). Then :

1. $N$ is a fuzzy seminorm and, for $0<\alpha<\beta<1$, we have $p_{\alpha} \leq q_{\alpha, N} \leq p_{\beta}$.

2. $p_{\alpha}=q_{\alpha, N}$, for all $\alpha$, iff $p_{\alpha}=\inf _{\beta>\alpha} p_{\beta}$.

3. $\tau_{N}$ is the topology generated by the seminorms $p_{\alpha}$.

4. $N$ is a fuzzy norm iff $\sup _{\alpha} p_{\alpha}(x)>0$ for all $x \neq 0$.

Proof. It is clear that $N(x)$ is increasing and $N(c x, t)=N\left(x,|c|^{-1} t\right)$ when $c \neq 0$. Let $N(x, t)>\alpha>0$, where $t>0$. There exists $\alpha<\beta<1$ such that $p_{\beta}(x)<t$. Let $0<s<t$ be such that $p_{\beta}(x)<s$. Then $N(x, s)>\beta>\alpha$, which proves that $N(x)$ is left continuous. Suppose now that $N(x, t) \wedge N(y, s)>\alpha>0$. There exists $\beta>\alpha$ such that $p_{\beta}(x)<t, p_{\beta}(y)<s$ and so $p_{\beta}(x+y) \leq p_{\beta}(x)+p_{\beta}(y)<t+s$, which implies that $N(x+y, t+s) \geq \beta>\alpha$. This proves that $N(x+y) \preceq N(x) \oplus N(y)$. Finally, $\lim _{t \rightarrow \infty} N(x, t)=1$. In fact, let $0<\alpha<1$ and $s>p_{\alpha}(x)$. For $t \geq s$ we have $N(x, t) \geq \alpha$, which proves our claim. So $N$ is a fuzzy seminorm. It is easy to see that $N$ is a fuzzy norm iff $\sup _{\alpha} p_{\alpha}(x)>0$ for all $x \neq 0$. Next we show that for $0<\alpha<\beta<1$, we have $p_{\alpha} \leq q_{\alpha, N} \leq q_{\beta}$. Indeed, if $q_{\alpha, N}(x)<t$, then $N(x, t)>\alpha$. There exists $\gamma>\alpha$ with $p_{\gamma}(x)<t$ and so $p_{\alpha}(x) \leq p_{\gamma}(x)<t$. This proves that $p_{\alpha} \leq q_{\alpha, N}$. Also, for $s>p_{\beta}(x)$, we have $N(x, s) \geq \beta>\alpha$ and hence $q_{\alpha, N}(x)<s$, which proves that $q_{\alpha, N}(x) \leq p_{\beta}(x)$. Thus

$$
p_{\alpha} \leq q_{\alpha, N} \leq \inf _{\beta>\alpha} p_{\beta} .
$$

(2) follows from (1) and from Lemma 3.4 while (3) follows from (1).

Example 3.9 Let $X$ be a topological space, $(E, N)$ a fuzzy normed space and $G=C_{b}(X, E)$ the space of all bounded continuous E-valued functions on $X$. For $f \in G$, we define

$$
N_{\infty}(f)=\bigvee_{x \in X} N(f(x)) .
$$

Then $N_{\infty}$ is a fuzzy norm on $G$ for which $\tau_{N_{\infty}}$ coincides with the topology of uniform convergence. 
Example 3.10 Let $\left(r_{n}\right)_{n=1}^{\infty}$ be an increasing sequence of continuous seminorms, on a locally convex space E, such that $\sup _{n} r_{n}(x)=\infty$ for each $x \neq 0$. Take $r_{0}=0$. For $0<\alpha<1$, there exists a unique positive integer $n$ such that

$$
(n-1) / n<\alpha<n /(n+1) \text {. }
$$

Take $p_{\alpha}=r_{n-1}$. Then $\left(p_{\alpha}\right)$ is an increasing family of continuous seminorms on E. Consider the fuzzy seminorm $N$ defined by

$$
N(x, t)=\sup \left\{\alpha: p_{\alpha}(x)<t\right\} .
$$

Then $\tau_{N}$ coincides with the topology generated by the seminorms $r_{n}$. This topology is clearly Hausdorff and thus $N$ is a fuzzy norm.

In the above example, for each $x \in E$ and each $t>0$, we have that either $N(x, t) \in\{0,1\}\}$ or $N(x, t)=n /(n+1)$ for some positive integer $n$. Indeed, assume that $N(x, t) \neq 0,1$. Then $x \neq 0$. There exists a unique positive integer $n$ such that $r_{n-1}(x)<t \leq r_{n}(x)$. Let $0<\alpha<1$. If $(n-1) / n<\alpha \leq n /(n+1)$, then $p_{\alpha}(x)=r_{n-1}(x)<t$. If $\alpha>n /(n+1)$ and $(m-1) / m<\alpha<m /(m+1)$, then $n \leq m-1$, which implies that $p_{\alpha}(x)=r_{m-1}(x) \geq r_{n}(x) \geq t$. It follows that $N(x, t)=n /(n+1)$ and so the claim is true. Let

$$
V=\{x: N(x, t) \geq(n+1) /(n+2)
$$

Using the claim we get that $V=\{x: N(x, t)>n /(n+1)\}$ and so $V$ is open. The set $V$ is not empty since it contains 0. If $r_{n-1} \neq 0$, then $V \neq E$. Indeed, assume that $V=E$. Then, for $\alpha=n /(n+1)$ and $y \in E=V$, we have $N(y, t)>\alpha$ and hence $q_{\alpha}(y)<t$. This, being true for all $y \in E, r_{n-1}=p_{\alpha} \leq q_{\alpha}=0$, a contradiction. Therefore, for $r_{n-1} \neq 0$, we have that $V$ is a non-empty proper subset of $E$ which is open and hence not closed since every non-trivial topological vector space is connected.

We will say that a topological vector space $E$ is fuzzy normable if there exists a fuzzy norm $N$ on $E$ such that $\tau_{N}$ coincides with the topology of $E$. A Hausdorff topological vector space $E$ is fuzzy normable iff it is locally convex and metrizable. Proof. We have shown that the condition is necessary. for the necessity, suppose that $E$ is locally convex and metrizable. Then, there exists an increasing sequence $\left(r_{n}\right)$ of continuous seminorms on $E$ such that, for each continuous seminorm $p$ on $E$ the exists an $n$ with $p \leq r_{n}$. As in the preceding example, there exists a fuzzy norm $N$ on $E$ such that $\tau_{N}$ coincides with the topology generated by the seminorms $r_{n}, n=1,2, \ldots$ This latter topology is the topology $9 \mathrm{f} E$.

\section{Some properties of fuzzy seminormed spaces}

Let $(E, N)$ be a fuzzy seminormed space and $A \subset E$. Then :

1. For a net $\left(x_{\delta}\right)$ in $E$, we have that $\lim _{\delta} N\left(x_{\delta}-x, t\right)=1$ for all $t>0$

2. $A$ is $\tau_{N^{-b o u n d e d}}$ iff $N(A)$ is bounded in $\mathcal{D}\left(R^{+}\right)$, which is equivalent to

$$
\lim _{t \rightarrow \infty} \inf _{x \in A} N(x, t)=1
$$

3. An element $x$ of $E$ belongs to the closure $\bar{A}$ of $A$ iff $\bigwedge_{y \in A} N(x-y)=\tilde{0}$, which is equivalent to $\sup \{N(x-y, t)$ : $y \in A\}=1$ for all $t>0$

4. $x$ belongs to the interior $A^{o}$ of $A$ iff $\bigwedge_{y \notin A} N(x-y) \neq \tilde{0}$, which is equivalent to $\sup _{y \notin A} N(x-y, t)<1$ for some $t>0$.

5. If $A$ is non-empty and proper subset of $E$, then

$$
\bigwedge_{x \in A, y \notin A} N(x-y)=\tilde{0},
$$

which is equivalent to

$$
\sup \{N(x-y, t): x \in A, y \notin A\}=1
$$

for each $t>0$. 
Proof. (1) Suppose that $x_{\delta} \rightarrow x$ and let $t>0$. The set $W_{t, \alpha}$ is a neighborhood of zero in $E$. Thus, there exists $\delta_{o}$ such that $x_{\delta}-x \in W_{t, \alpha}$, i.e. $N\left(x_{\delta}-x, t\right)>\alpha$, for all $\delta \geq \delta_{o}$, which proves that $\lim _{\delta} N\left(x_{\delta}-x, t\right)=1$. The converse follows analogously.

(2) Assume that $A$ is bounded and let $)<\alpha<1$. There exists $t>0$ such that $A \subset t W_{1, \alpha}$. Thus, for $x \in A$, we have $N(x, t)>\alpha$, which clearly proves that $\lim _{t \rightarrow \infty} \inf _{x \in A} N(x, t)=1$. The proof of the converse is analogous.

(3) Suppose that $x \in \bar{A}$ and let $t>0$. Given $0<\alpha<1$, there exists $y \in A$ such that $x-y \in W_{t, \alpha}$, i.e. $N(x-y, t)>\alpha$. Hence $\sup _{y \in A} N(x-y, t)=1$. The converse follows in an analogous way.

(4) Assume that $x \in A^{o}$. Since $A^{o}$ is the complement of the closure $\overline{A^{c}}$ of $A^{c}=E \backslash A$, we have that $x \notin \overline{A^{c}}$. In view of (3), there exists $t>0$ such that

$$
\sup _{y \notin A} N(x-y, t)<1 .
$$

The converse again follows from (3).

(5) Assume that $A \neq \emptyset, E$. If $\partial A$ is the boundary of $A$, then $\bar{A}=A^{o} \cup \partial A$. Now $\partial A$ cannot be empty. Indeed, if $\partial A$ were empty, then $A=\bar{A}=A^{o}$ and so $A$ would be both open and closed which cannot be true since $E$ is connected. Let now $z \in \partial A=\bar{A} \cap \overline{A^{c}}$. Since $z \in \bar{A}$, there exists by (3) an $x \in A$ such that $N(x-z, t / 2)>\alpha$. Similarly, since $z$ belongs to the closure of $A^{c}$, there exists $y \notin A$ such that $N(z-y, t / 2)>\alpha$. Now

$$
N(x-y, t) \geq N(x-z, t / 2) \wedge N(z-y, t / 2)>\alpha,
$$

which completes the proof.

Theorem 4.1 For a non-empty subset $A$ of a seminormed space $(E, N)$, the following are equivalent:

1. A is totally bounded.

2. Given $t>0$, there exist $x_{1}, \cdots, x_{n}$ in $A$ such that

$$
\text { inf } f_{x \in A} \max _{1 \leq k \leq n} N\left(x-x_{k}, t\right) \geq \alpha .
$$

3. Given $t>0$, there exist $x_{1}, \cdots, x_{n}$ in $E$ such that

$$
i n f_{x \in A} \max _{1 \leq k \leq n} N\left(x-x_{k}, t\right) \geq \alpha .
$$

Proof. (1) $\Rightarrow(2)$ Suppose that $A$ is totally bounded and let $t>0,0<\alpha<1$. The set $W_{t, \alpha}$ is a neighborhood of zero and hence there exists a finite subset $S=\left\{x_{1}, \cdots, x_{n}\right\}$ in $A$ such that $A \subset S_{+} W_{t, \alpha}$. If now $x \in A$, then $x-x_{k} \in W_{t, \alpha}$, for some $1 \leq k \leq n$, and hence $N\left(x-x_{k}, t\right)>\alpha$. This proves that $\inf _{x \in A} \max _{1 \leq k \leq n} N\left(x-x_{k}, t\right)>\alpha$.

$(3) \Rightarrow(1)$ Let $t>0$ and $0<\alpha<1$. Let $0<\alpha<\beta<1$. By (3), there are $x_{1} \cdots, x-n$ in $E$ such that $\max _{1 \leq k \leq n} N\left(x-x_{k}, t\right) \geq \beta$ for all $x \in A$. Hence, given $x \in A$, there exists $k$ such that

$$
x-x_{k} \in V=\{y: N(y, t) \geq \beta\} \subset W_{t, \alpha} .
$$

Thus $A \subset\left\{x_{1}, \cdots, x_{n}\right\}+W_{t, \alpha}$, which completes the proof.

Theorem 4.2 For a fuzzy normed space $(E, N)$, the following are equivalent

1. There exists $0<\alpha<1$ such that $\lim _{t \rightarrow \infty} \inf \{N(x, t): N(x, t)>\alpha\}=1$

2. There exists $0<\alpha<1$ such that $\tau_{N}$ is generated by the seminorm $q_{\alpha, N}$ (in this case $q_{\alpha, N}$ is a norm).

Corollary 4.3 If $(E, N)$ is finite dimensional fuzzy normed space, then there exists $0<\alpha<1$ such that

$$
\lim _{t \rightarrow \infty}\{N(x, t): N(x, t)>\alpha\}=1 .
$$

Proof The topology $\tau_{N}$ is Hausdorff. But every finite dimensional Hausdorff topological vector space is normable. Hence the result follows from the preceding theorem.

Lemma 4.4 Let $f$ be a linear functional on a fuzzy seminormed space $(E, N)$. For $t>0$ and $0<\alpha 1$, the following are equivalent :

1. If $x \in E$ and $q_{\alpha}(x)<1$, then $|f(x)|<1$. 
2. $N(x, t \mid f(x)) \leq \alpha$ for all $x \in E$.

Proof . (1) $\Rightarrow(2)$. Clearly $N(x, t \mid f(x)) \leq \alpha$ if $f(x)=0$. Suppose that $f(x) \neq 0$ and let $y=f(y)$. Then $|f(y)|=1$. By our hypothesis (1) we have that $q_{\alpha}(y) \geq t$, i.e. $q_{\alpha}(x) \geq t|f(x)|$, which proves that $N(x, t \mid f(x)) \leq \alpha$ (by lemma 2.6))

$(2) \Rightarrow(1)$. If $q_{\alpha}(x)<t$, then $N(x, t)>\alpha$, which (by our hypothesis (2)) implies that $t|f(x)|, t$, i.e. $|f(x)|<1$, and the lemma follows.

Theorem 4.5 A linear functional $f$ on a fuzzy seminormed space $(E, N)$ is continuous iff there exists $t>0$ such that

$$
\sup \{N(x, t|f(x)|) x \in E\}<1 \text {. }
$$

Hence $f$ is continuous iff there are $t>0$ and $0<\alpha<1$ such that $N(x, t|f(x)|) \leq \alpha$ for all $x \in E$

Proof. Suppose That $\mathrm{f}$ is continuous. Then, there exist $0<\alpha<1$ and $0<t|f(x)|<1$ when $q_{\alpha}(x)<t$. Then

$$
\sup \{N(x, t|f(x)|) x \in E\}<1
$$

Conversely, let $t>0$ be such that

$$
\sup _{x \in E} N(x, t|f(x)|)<\alpha<1
$$

By the preceding lemma, $|f(x)|<1$ when $q_{\alpha}(x)<t$ and so $\mathrm{f}$ is continuous. The result now clearly follows.

With an analogous proof we prove the following

Theorem 4.6 Let $(E, N)$ be a fuzzy seminormed space and $F$ be a locally convex space. Then a linear $T:(E, N) \rightarrow$ $f$ is continuous iff, for each continuous seminorm $p$ on $F$ there exists $t>0$ there exists $0<\alpha<1$ such that $N(x, t p(T x)) \leq \alpha$ for all $x \in E$.

We also have the following

Theorem 4.7 Let $(E, N)$ be a fuzzy seminormed space and let $H$ be a subset of the dual space of $E$. Then $H$ is equicontinuous iff there exists $t>0$ such that

$$
\sup \{N(x, t|f x|): x \in E . f \in H\}<1 .
$$

Theorem 4.8 Let $N_{1}, N_{2}, \cdots, N_{n}$ be fuzzy seminorms on a vector space E. For $\left.x \in E\right)$ define $N(x)=\oplus_{k=1}^{n} N_{k}(x)$. Then $N$ is a fuzzy seminorm and $q_{\alpha, N}=\sum_{k=1}^{n} q_{\alpha, N_{k}}$.

Proof. It easy to show that $\mathrm{N}$ is a fuzzy seminorm. Suppose that $q_{\alpha, N}(x)>t$. Then $N(x, t)>\alpha$. There are $t_{k}>0, \sum_{k=1}^{n} t_{k}=t$, such that $\min _{1 \leq k \leq n} N_{k}\left(x, t_{k}\right)>\alpha$. Then $q_{\alpha, N_{k}}(x)<t_{k}$ and $\sum_{k=1}^{n} q_{\alpha, N_{k}}(x)<t$. This proves that $q_{\alpha, N}=\geq \sum_{k=1}^{n} q_{\alpha, N_{k}}=d$. On the other hand let $d<s$. There are $s_{k}>0$ such that $q_{\alpha, s_{k}}(x)<s_{k}, \sum_{k=1}^{n} s_{k} s$. Then $N_{k}\left(x_{k}\right)>\alpha N(x, t) \geq \wedge_{k=1}^{n} N_{k}\left(x, s_{k}\right)>\alpha$, which implies that $q_{\alpha, N}(x)<d$. This proves that $q_{\alpha, N}(x) \leq d$ and the result follows.

Theorem 4.9 (Hahn Banach) Let $F$ be a subspace of a fuzzy norm space $(E, N)$ and let $f$ be a linear functional on $F$ such that, for so $t>0$ and some $0<\alpha<1$ we have $N(x, t|f(x)|) \leq \alpha$ for all $x \in F$. Then there exists a linear extension $g$ of $f$ such that $N(x, t|g(x)|) \leq \alpha$ for all $x \in E$.

Proof. By lemma 3.5 our hypopthesis is equivalent to $|f(x)|<1$ when $q_{\alpha}(x)<1$. Set

$$
\|f\|_{\alpha}=\sup \left\{|f(x)|: x \in F, q_{\alpha}(x) \leq 1\right\}=\sup \left\{|f(x)|: q_{\alpha}(x)<1\right\} \leq t^{-1}
$$

Thus $|g(x)| \leq t^{-1} q_{\alpha}(x)$ for all $x \in F$. By the Hahn Banach Theorem there exists a linear extension $\mathrm{g}$ of $\mathrm{f}$ such that $|g(x)| \leq t^{-1} q_{\alpha}(x)$ for all $x \in E$. If now $x \in E$ and $q_{\alpha}(x)<t$, then $|g(x)|<1$. In view of lemma 3.5 we get $N(x, t|g(x)|) \leq \alpha$ for all $x \in E$.

Next we give a fixed point theorem analogous to the one that holds for metric spaces. We will need the following

Lemma 4.10 If $\xi \in \mathcal{D}\left(\mathcal{R}^{+}\right)$is such that $\xi c \xi$ for some $0<c<1$, then $\xi=\tilde{0}$. Also $\left(c_{1} \xi\right) \oplus\left(c_{2} \xi\right)\left(c_{1}+c_{2}\right) \xi$. 
Proof. By induction we have that $\xi c^{n} \xi$ for each positive integer n. For $t>0 \xi(t) \geq \lim \xi\left(c^{-n} t\right)=1$. Thus $\xi(t)=1$ for all $t>0$ and hence $\xi=\tilde{0}$. Let $s>0, s=s_{1}+s_{2}$ and $c=c_{1}+c_{2}$. We cannot have that $s_{1} / c_{1}, s_{2} / c_{2}>s / c$. Thus

$$
(c \xi)(s) \geq \xi\left(s_{1} / c_{1}\right) \wedge \xi\left(s_{2} / c_{2}\right)=\left(c_{1} \xi\right)\left(s_{1}\right) \wedge\left(c_{2} \xi\right)\left(s_{2}\right) .
$$

Thus $c_{1} \xi \oplus c_{2} \xi c \xi$

Definition 4.11 A fuzzy Banach space is a fuzzy normed space $(E, N)$ for which $\left(E, \tau_{N}\right)$ is complete.

Theorem 4.12 (A fixed point theorem) Let $(E, N)$ be a fuzzy Banach space and let $f: E \rightarrow E$ be a function for which there exists $0<c<1$ such that $N(f(x)-f(y)) c N(x-y))$ for all $x, y \in E$. Then $f$ has a unique fixed $x_{1}$. Moreover if $x_{1}$ is any element of $E$ and $x_{n+1}=x_{n}$, then $\left(x_{n}\right)$ converges to $x_{1}$.

Proof.The proof is analogous to the one for complete metric spaces. First observe that $\mathrm{f}$ is uniformly continuous. Indeed, given $t>0$ and $0<\alpha<1$, take $s=c^{-1} t$. If $x-y \in W_{s, \alpha}$, then

$$
N(f(x)-f(y), t) \geq N(x-y, s)>\alpha
$$

which proves that $\mathrm{f}$ is uniformly continuous. Now by induction we get that

$$
N\left(x_{n+1}-x_{n}\right) N\left(x_{2}-x_{1}\right)=c^{n-1} \xi .
$$

For $m>n$, we have

$$
\left.N\left(x_{m}-x_{n}\right) \oplus_{k=n}^{m-1} N\left(x_{k+1}-x_{k}\right) \oplus_{k=n}^{m-1} c^{k-1} \xi\right)\left(c^{n-1}+\cdots c^{m}\right) \xi c^{n-1} /(1-c) \xi .
$$

It follows that $\left(x_{n}\right)$ is Cauchy and thus $x_{n} x_{o}$ for some $x_{o}$. Since $\mathrm{f}$ is continuous, we have that $f\left(x_{n}\right) \rightarrow f\left(x_{o}\right)$. But $f\left(x_{n}\right) \rightarrow x_{o}$. Hence $f\left(x_{o}\right)=x_{o}$, i.e. $x_{o}$ is a fixed point. Finally, suppose that $x, y$ are fixed points for $\mathrm{f}$ and let $\eta=N(x-y)$. Then $\eta N(f(x)-f(y)) c N(x-y))=c \xi$ and hence $\eta=\tilde{0}$, by the preceding lemma. It follows that $x-y=0$ since $N$ is a fuzzy norm which competes the proof.

Theorem 4.13 For a sequence $\left(x_{n}\right)$, in a fuzzy seminormed space $(E, N)$, the following are equivalnet:

1. $\xi=\wedge_{n} \oplus_{k=1}^{n} N\left(x_{k}\right)$ exists in $\mathcal{D}\left(R^{+}\right)$.

2. For each $0<\alpha<1$ we have that $\sum_{n=1}^{\infty} q_{\alpha}\left(x_{n}\right)<\infty$.

Proof . (1) $\Rightarrow(2)$. Let $0<\alpha<1$. There exists $t>0$ such that $\xi(t)>\alpha$. There exists $0<s<t$ such that $\eta_{n}(s)>\alpha$, for all $\mathrm{n}$, where $\eta_{n}=\oplus_{k=1}^{n} N\left(x_{k}\right)$ Given $\mathrm{n}$, there exist $s_{k}$ such that $\sum_{1}^{n} s_{k}=s$ and $N\left(x_{k}, s_{k}\right)>\alpha$. Hence $q_{\alpha}\left(x_{k}\right)<s_{k}$ and so $\sum_{1}^{n} q_{\alpha}\left(x_{k}\right)<s<t$. This proves that $\sum_{n=1}^{\infty} q_{\alpha}\left(x_{n}\right) \leq t$.

$(2) \Rightarrow(1)$. It suffices show that, for $\eta_{n}=\oplus_{k=1}^{n} N\left(x_{k}\right)$, we have that

$$
\lim _{t \rightarrow \infty} \inf _{n} \eta_{n}(t)=1 .
$$

So, let $0<\alpha<1$. By our hypothesis (2), there exists $\mathrm{s}$ such that

$$
\sum_{1}^{\infty} q_{\alpha}\left(x_{k}\right)<s<\infty
$$

For a given $\mathrm{n}$, there exist $s_{1}, \cdots, s_{n}$ such that $q_{\alpha}\left(x_{k}\right)<s_{k}$ and $\sum_{1}^{n} s_{k}<s$. Now $N\left(x_{k}, s_{k}\right)>\alpha$ and hence

$$
\eta_{n}(s) \geq \bigwedge_{k=1}^{n} N\left(x_{k}, s_{k}\right)>\alpha .
$$

Thus $\inf _{n} \eta_{n}(s) \geq \alpha$ which proves that $\lim _{t \rightarrow \infty} \eta_{n}(t)=1$. This completes the proof.

Theorem 4.14 For a sequence $\left(x_{n}\right)$, in a fuzzy seminormed space $(E, N)$, the following are equivalent :

1. The $\sup _{n} \wedge \bigoplus_{k=1}^{n} N\left(x_{k}\right)$ exists in $\mathcal{D}\left(R^{+}\right)$.

2. For each $0<\alpha<1$ we have that $\sum_{n=1}^{\infty} q_{\alpha}\left(x_{n}\right)<\infty$. 
Proof. $(1) \rightarrow(2)$. Let $\xi=\bigwedge_{n} \bigoplus N\left(x_{k}\right.$ and $0 \alpha<1$. There exists $t$ such that $\inf _{n}\left[\bigoplus_{k=1}^{n} N\left(x_{k}\right)\right]>\alpha$. Given n, there are $t_{1}, \cdots, t_{n}$ such that $\sum_{1}^{n} t_{k}=t$ and $N\left(x_{k}, t_{k}\right)>\alpha$ for all n. Now $q_{\alpha}\left(x_{k}\right)<t_{k}$ and so $\sum_{1}^{n} q_{\alpha}\left(x_{k}\right) \leq t$. This proves that $\sum_{1}^{\infty} q_{\alpha}\left(x_{k}\right) \leq t$.

$(2) \Rightarrow(1)$. Assume that $\sup _{n} \bigwedge \bigoplus_{k=1}^{n} N\left(x_{k}\right)$. There exists $0<\alpha<1$ such that

$$
\sup _{t}\left[\inf _{n} \oplus_{1}^{n} N\left(x_{k}\right)\right](t)<\alpha .
$$

Choose $t>\sum_{1}^{\infty} q_{\alpha}\left(x_{n}\right)$. There exists a sequence $\left(t_{n}\right)$ such that $\sum t_{n}<t$ and $q_{\alpha}\left(x_{n}\right)<t_{n}$ for all n. Now, for each $\mathrm{n}$,

$$
\left[\bigoplus_{k=1}^{n} N\left(x_{k}\right)\right](t) \geq \bigwedge_{k=1}^{n} N\left(x_{k}, t_{k}\right)>\alpha .
$$

This roves that

$$
\sup _{t>0} \inf _{n}\left[\bigoplus_{1}^{n} N\left(x_{k}\right](t)=1\right.
$$

and so $\bigwedge_{n} \bigoplus_{k=1}^{n} N\left(x_{k}\right.$ exists.

Lemma 4.15 For a metrizable locally convex space $E$ the following are equivalent:

1. If $\left(x_{n}\right)$ is a sequence in $E$ such that $\sum_{n=1}^{\infty} p\left(x_{n}\right)<\infty$ for every continuous seminorm $p$ on $E$, then the series $\sum_{n=1}^{\infty} x_{n}$ is convergent.

2. E is complete.

Proof . $(2) \Rightarrow(1)$. Suppose that the condition for a sequence $\left(x_{n}\right)$ in $E$. L:et $y_{n}=\sum_{k=1}^{n} x_{k}$. For $p \in c s(E)$ and $m>n$, we have that $p\left(y_{m}-y_{n}\right) \leq \sum_{n+1}^{m} p\left(x_{k}\right) \rightarrow 0$ when $n \rightarrow \infty$. Thus $\left(y_{n}\right)$ is is Cauchy and hence convergent. $(1) \Rightarrow(2)$. Since $E$ is metrizable, there exists an increasing sequence $\left(p_{n}\right)$ of continuous seminorms on $E$ such that, for each $p \in c s(E)$, the exists an $\mathrm{n}$ such that $p \leq p_{n}$. Let $\left(x_{n}\right)$ be a Cauchy sequence $E$. Choose an indices $n_{1}<n_{2}<\cdots$ such that $p_{k}\left(x_{n}-x_{m}\right)<1 / 2^{k}$ for all $n, m>n_{k}$. Consider the series Given $p \in c s(E)$, choose $k_{o}$ such that $p \leq k_{o}$. Then

$$
\sum_{k=1}^{\infty} p\left(y_{k}=\sum_{k=1}^{k_{o}} p\left(y_{k}\right)+\sum_{k>k_{o}} p\left(y_{k}\right) .\right.
$$

But for $k>k_{o}$, we have $p\left(y_{k}\right) \leq p_{k}\left(y_{k}\right)<1 / 2^{k}$. Thus

$$
\sum p\left(y_{k}\right) \leq \sum_{k=1}^{k_{o}} p\left(y_{k}\right)+\sum_{k>k_{o}} 2^{-k}<\infty .
$$

Now by our hypothesis the series $\sum y_{n}$ converges in $E$. For each $\mathrm{m}$ we have $z_{m}=\sum_{k=}^{m} y_{k}=x_{n_{m}}$. So $\left(z_{m}\right)$ is a convergent subsequence of $\left(x_{n}\right)$. Since $\left(x_{n}\right)$ is Cauchy, it follows that it converges and the proof is complete.

Theorem 4.16 For a fuzzy normed space $(E, N)$, the followig are equivalent:

1. $(E, N)$ is complete.

2. For every sequence $\left(x_{n}\right)$ in $E$, for which the element $\xi=\bigvee_{n} \bigoplus_{k=1}^{n} N\left(x_{k}\right)$ exists in $\mathcal{D}\left(R^{+}\right)$, the series $\sum x_{n}$ converges in $E$.

Proof. For each $\tau_{N}$ continuous seminorm there exists $0<\alpha<1$ and $c>0$ such that $p \leq c p_{\alpha}$. Now result follows from the preceding lemma and the theorem 3.15 .

\section{Subspaces, product and quotient spaces}

let $F$ be a subspace of a fuzzy seminormm space. Define $N_{F}$ to be the restriction of $N$ to $F$. Then $N_{F}$ is a fuzzy seminorn on $F$. Meoreover, For $0<\alpha<1$ and $x \in F$, we have

$$
q_{\alpha, N_{F}}(x) \inf \left\{t: t>0, N_{F}(x, t)>\alpha\right\}=\inf \{t: N(x, t)>\alpha\}=q_{\alpha, N}(x) .
$$

Hence the topology induced on $F$ by the seminorm $N_{F}$ coincides with the topology of $F$ as a subspace of $\left(E, \tau_{N}\right)$. 
Definition 5.1 A fuzzy seminorm $N$, on a topological vector space $E$, is said to be continuous if the induced topology $\tau_{N}$ is coarser than the topology of $E$.

It is well known that, if $F$ is subspace of $E$, then each continuous seminorm on $F$ has a continuous extension on all $\mathrm{f} E$. In the next theorem we will show that the same happens for fuzzy continuous seminorms.

Theorem 5.2 Let $F$ be a subspace of a locally convex space $E$ and let $N$ be a continuous fuzzy fuzzy seminorm on F. Then:

1. There exists a continuous fuzzy seminorm $N^{\prime}$ on $E$ such that $N=N^{\prime}$ on $F$.

2. For each $0<\alpha<1$ we have that $q_{\alpha, N}(x)=q_{\alpha, N^{\prime}}(x)$ for $x \in F$.

3. If $F$ is dense, then $N^{\prime}$ is unique.

Proof. Let $\alpha_{o}=0<\alpha_{1}<\cdots<\alpha_{n} \uparrow 1$. For $0<\alpha<1$, let $q_{\alpha}=q_{\alpha, N}$. Let $p_{o}$ be the zero seminorm on $E$. For $\mathrm{n}$ a positive integer, there exists a continuous seminorm $r_{n}$ on $E$ such that $r_{n}=q_{\alpha_{n}}$ on $F$. Let $p_{n}=r_{1} \vee \cdots \vee r_{n}$. Then $p_{n}$ is a continuous seminorm on $E$. For $x \in F$, we have

$$
\left.p_{n}(x)=\max _{1 \leq k \leq 1} r_{k}(x)=\max _{1 \leq k \leq n} q_{\alpha_{k}}(x)=q_{\alpha_{n}} x\right) .
$$

Also $p_{n} \leq p_{m}$ if $n \leq m$. For $0<\alpha<1$, there exists a unique $m$ such that $\alpha_{m-1}<\alpha \leq \alpha_{m}$. Define

$$
\sigma_{\alpha}(x)=\inf \left\{q_{\alpha}(y)+p_{m}(x-y): y \in F\right\}
$$

for $x \in E$. Then $\sigma_{\alpha}$ is a seminorm on $E$. Indeed, $\sigma_{\alpha}(0) \leq q_{\alpha}(0)+p_{m}(0)=0$. For $c \neq 0$, we have $\sigma_{\alpha}(x)=\inf \left\{q_{\alpha}(y)+p_{m}(c x-y): y \in F\right\}=\inf \left\{q_{\alpha}(c y)+p_{m}(c x-c y): y \in F\right\}$

$=|c| \inf \left\{q_{\alpha}(y)+p_{m}(x-y): y \in F\right\}=|c| \sigma_{\alpha}(x)$.

To prove that the triangle inequality, let $t_{1}>\sigma_{\alpha}(x), t_{2}>\sigma_{\alpha}(z)$. There are $y_{1}, y_{2}$ in $F$ such that $q_{\alpha}\left(y_{1}\right)+p_{m} \in F$ and so

$$
\sigma_{\alpha}(x+z) \leq q_{\alpha}(y)+p_{m}(x+z-y) \leq q_{\alpha}\left(y_{1}\right)+q_{\alpha}\left(y_{2}\right)+p_{m}\left(x-y_{1}\right)+p_{m}\left(z-y_{2}\right)<t_{1}+t_{2} .
$$

This shows that $\sigma_{\alpha}(x+z) \leq \sigma_{\alpha}(x)+\sigma_{\alpha}(z)$ and therefore $\sigma_{\alpha}$ is a seminorm on $E$. Moreover $\sigma_{\alpha}(x) \leq q_{\alpha}(0)+p_{m}(x)$ and hence $\sigma$ is continuous. For $y \in F$, we have

$$
q_{\alpha}(x) \leq q_{\alpha}(y)+q_{\alpha}(x-y) \leq q_{\alpha}(y)+q_{\alpha_{m}}(x-y)=q_{\alpha}(y)+p_{m}(x-y) .
$$

Thus $q_{\alpha}(x) \leq \sigma_{\alpha}(x)$. On the other hand, $\sigma_{\alpha}(x) \leq q_{\alpha}(x)+p_{m}(o)=q_{\alpha}(x)$ and hence $\sigma_{\alpha}(x)=q_{\alpha}(x)$. For $\alpha \leq \beta$, we have that $\sigma_{\alpha} \leq \sigma_{\beta}$. Indeed, let $\alpha_{m-1}<\alpha \leq \alpha_{m}, \alpha_{n-1}<\beta \leq \alpha_{n}$. Then $\alpha_{m-1}<\alpha \leq \beta \leq \alpha_{n}$ and so $m-1<n$, which implies that $m \leq n$ and therefore $\sigma_{\alpha} \leq \sigma_{\beta}$. Now define, for $\in E, N^{\prime}(x, t)=\sup \left\{\alpha: \sigma_{\alpha}(x)<t\right\}$. Then $N^{\prime}$ is a fuzzy seminorm on $E$. For $0<\alpha<1$, we have

$$
\sigma_{\alpha}(x) \leq q_{\alpha, N^{\prime}}(x) \leq \sigma_{\beta}(x) .
$$

Thus $q_{\alpha, N^{\prime}}$ is continuous on $E$ and hence $N^{\prime}$ is continuous. Moreover $N(x)=N^{\prime}(x)$ for $x \in F$. Indeed, for $x \in F$, we have

$$
N^{\prime}(x, t)=\sup \left\{\alpha: \sigma_{\alpha}(x)<t\right\}=\sup \left\{\alpha: q_{\alpha}(x)<t\right\}=N(x, t) .
$$

Thus $N=N^{\prime}$ on $f$. As we have seen in the beginning of the section we have that $q_{\alpha, N)=q_{\alpha}, N^{\prime}}$ on $F$. Finally, suppose that $F$ is dense and let $N_{1}, N_{2}$ be continuous extensions of $N$. Since $q_{\alpha, N_{1}}, q_{\alpha, N_{2}}$ are ontinuous on $E$ we have that $q_{\alpha, N_{1}}=q_{\alpha, N_{2}}$ which proves that $N_{1}=N_{2}$. This complets the proof.

Theorem 5.3 Let $E, F$ be linear spaces and let $T: E \rightarrow F$ be a linear map. Let $N$ be a fuzzy seminorm on $F$. Define

$$
N^{\prime}=T^{-1}(N): E \rightarrow \mathcal{D}\left(R^{+}\right), N^{\prime}(x)=N(T x) .
$$

Then $N^{\prime}$ is a fuzzy seminorm on $E$ and $q_{\alpha, N^{\prime}}=T^{-1}\left(q_{\alpha, N}\right)$.

Proof . It is easy to see that $N^{\prime}$ is a fuzzy seninorm.Moreover, for $x \in E$,

$$
\left.q_{\alpha, N^{\prime}}(x)=\inf \left\{t: N^{\prime}(x, t)>\alpha\right\}=\inf \right\}\{t: N(T x, t)>\alpha\}=q_{\alpha . N}(T x) .
$$


Corollary 5.4 If $E$ is a topological vector space, $T: E \rightarrow F$ a linear map and $N$ a fuzzy seminorm on $F$, then $T: \rightarrow\left(F, \tau_{N}\right)$ is continuous iff $T^{-1}(N)$ is a continuous seminorm on $E$.

Theorem 5.5 Let $(E, N)$ a fuzzy seminorm space and $F$ a subspace Define

$$
N_{o}: E / F \rightarrow \mathcal{D}\left(R^{+}\right), N_{o}(x+F)+\bigwedge_{y \in F} N(x+y) / .
$$

Then

1. $N_{o}$ is a a fuzzy seminorm on $E / F$.

2. For $0<\alpha<1$, we have $q_{\alpha, N_{o}}(x+F)+\inf _{y \in F} q_{\alpha, N}(x+y)$.

3. $N_{o}$ is a fuzzy norm iff $F$ is $\tau_{N}$-closed in $E$.

Proof . (1). For $t>0$, we have $N_{o}(x+F, t)=\sup _{y \in F} q_{\alpha, N}(x+y)$. It is easy to see that, for $c \neq 0$, we have

$$
N_{o}(x+F, t)=N_{o}(x+F, t /|c|) .
$$

If

$$
N_{o}\left(x+F, t_{1}\right) \wedge N_{o}\left(y+F, t_{2}\right)>\alpha>0,
$$

there exist $y_{1}, y_{2} \in F$ such that $N\left(x+y_{1}, t_{1}\right) \wedge N\left(y+y_{2}, t_{2}\right)>\alpha$ and so

$$
N_{o}\left(x+y+F, t_{1}+t_{2}\right) \geq N\left(x+y+y_{1}+y_{2}, t_{1}+t_{2}\right) \geq N\left(x+y_{1}, t_{1}\right) \wedge N\left(y+y_{2}, t_{2}\right)>\alpha,
$$

which proves that $N_{o}(x+y+F) N_{o}(x+F) \oplus N_{o}(y+F)$. So $N_{o}$ is a fuzzy seminorm. Next we show that

$$
q_{\alpha, N_{o}}(x+F)=\inf _{y \in F} q_{\alpha, N}(x+y) .
$$

Indeed, if $q_{\alpha, N_{o}}(x+F)<t$, then $N_{o}(x+F, t)>\alpha$ and hence $N(x+y, t)>\alpha$, for some $y \in F$, which implies that $q_{\alpha, N}(x+y)<t$. This proves tha

$$
d=\inf _{y \in F} q_{\alpha, N}(x+y) \leq q_{\alpha, N_{o}}(x+F) .
$$

On the other hand, let $t>d$. There exists $y \in F$ with $q_{\alpha, N}(x+y)<t$ and so $N(x+y, t)>\alpha$. Therefore

$$
N_{o}(x+F) \geq N(x+y, t)>\alpha
$$

which implies that $q_{\alpha, N_{o}}(x+F)<t$. This proves that $q_{\alpha, N_{o}}(x+F) \leq d$. Finally, if $F$ is closed and $x \in \bar{F}=F$, then(by theorem 3.1) there exists $t>0$ such that

$$
N_{o}(x+F)=\sup \{N(x-y, t): y \in F\}>0,
$$

which implies $N_{o}(x+F) \neq \tilde{0}$ and so $N_{o}$ is a fuzzy norm. Conversely assume that $N_{o}$ is a fuzzy norm. Then, given $x \notin F$ there exists $0<\alpha<1$ such that $q_{\alpha, N_{o}}(x+F)>t>0$. Now

$$
\sup _{y \in F} N(x-y, t)=N_{o}(x+F, t) \leq \alpha<1
$$

and hence $x \notin \bar{F}$, which proves that $F$ is closed. This completes the proof.

Theorem 5.6 Let $\left(E_{k}, N_{k}\right), k=1, \cdots, n$, be a finite family of fuzzy seminormed spaces and let $E=\prod_{k=1}^{n} E_{k}$. For $x=\left(u_{1}, \cdots, u_{n}\right)$ in $E$, we define

$$
N(x)=\oplus_{k=1}^{n} N_{k}\left(u_{k}\right) .
$$

Then:

1. $N$ is a fuzzy seminorm on $E$.

2. $q_{\alpha, N}(x)=\sum_{k=1}^{n} q_{\alpha, N_{k}}\left(u_{k}\right)$.

3. $t_{N}$ is the product topology. 
4. $N$ is a fuzzy norm iff each $N_{k}$ is a fuzzy norm.

Proof. Using an argument analogous to the one used in theorem 3.9, we get that $N$ is a fuzzy seminorm and that $q_{\alpha, N}(x)=\sum_{k=1}^{n} q_{\alpha, N_{k}}\left(u_{k}\right)$. Assume that each $N_{k}$ is a norm. If $x=\left(u_{1}, \cdots, u_{n}\right)$ is not the zero element, then $u_{k} \neq 0$ for some $k$. Since $N_{k}$ is a norm, $q_{\alpha, N_{k}}\left(u_{k}\right) \neq 0$, for some $0<\alpha<1$, and so $q_{\alpha, N}(x) \neq 0$, which proves that $N$ is a fuzzy norm. On the other hand, suppose that some $N_{j}$ is not a fuzzy norm. There exists $u \neq 0$ such that $N_{j}(u)=\tilde{0}$. Let $x=\left(u_{1}, \cdots, u_{n}\right)$ with $u_{k}=0$, for $k \neq j$, and $u_{j}=u$. Then $N(x)=\tilde{0}$ and hence $N$ is not a fuzzy norm. Since $\tau_{N}$ is clearly the product topology, the result follows.

Theorem 5.7 Let $\left(E_{n}, N_{n}\right)$ be a sequence of fuzzy seminorm spaces and let $E=\prod_{n=1}^{\infty} E_{n}$. For $0<\alpha<1$. define $r_{\alpha, n}(x)=\sum_{k=1}^{n} q_{\alpha, N_{k}}\left(u_{k}\right)$ for $x=\left(u_{k}\right) \in E$. Let $\alpha_{n}=n /(n+1)$. For $1 /(n+1) \leq 1-\alpha<1 / n$, take $p_{\alpha}=r_{\alpha_{n}, n}$. Then $\left\{p_{\alpha}: 0<\alpha<1\right\}$ is an increasing family of fuzzy seminorms on $E$. If

$$
N(x, t)=\sup \left\{\alpha: p_{\alpha}(x)<t\right\}
$$

(the supremum over the empty family is taken to be zero), then :

1. $N$ is a fuzzy seminorm on $E$.

2. $N(x, t)=\sup \left\{n /(n+1): r_{\alpha_{n}, n}(x)<t\right\}=\sup \left\{n /(n+1):\left[\bigoplus_{k=1}^{n} N_{k}(k)\right](t)>n /(n+1)\right\}$.

3. $\tau_{N}$ coincides with the product topology.

4. $N$ is a fuzzy norm iff each $N_{k}$ is a fuzzy norm.

Proof. It is clear is a seminorm on $E$. If $0<\alpha<\beta<1,(n-1) / n<\alpha \leq n /(n+1)$ and $(m-1) / m<\beta \leq m /(m+1)$, then $n \leq m$ and hence $\alpha_{n} \leq \alpha_{m}$, which implies that

$$
p_{\alpha}=r_{\alpha_{n}, n} \leq r_{\alpha_{m}, m}=p_{\beta} .
$$

It follows that $N$ is a fuzzy seminorm on $E$. Since for each $0<\alpha<1$ we have hat $p_{\alpha}=r_{\alpha_{n}, n}$ for some $\mathrm{n}$, it follows that

$$
N(x, t)=\sup \left\{n /(n+1): r_{\alpha_{n}, n}<t\right\} .
$$

Claim. $r_{\alpha_{n}, n}(x) \Leftrightarrow\left[\bigoplus_{k=1}^{n} N_{k}\left(u_{k}\right)\right](t)>n /(n+1)$.

Indeed, suppose that $r_{\alpha_{n}, n}(x)_{<}$. Then there are $t_{k}, q_{\alpha_{n}, n}\left(u_{k}\right)<t_{k}$ and $\sum_{k=1}^{n} t_{k}<t$. Hence $N_{k}\left(u_{k}\right)>\alpha_{n}$, for $k=1, \cdots, n$, and so

$$
\left[\bigoplus_{k=1}^{n} N_{k}\left(u_{k}\right)\right](t) \geq \bigwedge N_{k}\left(u_{k}, t_{k}\right)>\alpha_{n} .
$$

Conversely, assume that $\left[\oplus_{k=1}^{n} N_{k}\left(u_{k}\right)\right](t)>\alpha_{n}$. There are $t_{k}, \sum_{k=1}^{n} t_{k}=t, N_{k}\left(u_{k}, t_{k}\right)>\alpha_{n}$. Thus $q_{\alpha_{n}, N_{k}}\left(u_{k}\right)<t_{k}$ and therefore $r_{\alpha_{n}, n}(x)<\sum_{k=1}^{n} t_{k}=t$. Hence (2) follows.

(3). Let $\left(x^{\delta}\right)_{\delta \in \Delta}$ be a net in $E, x^{\delta}=\left(u_{\delta_{k}}\right)$. If $x^{\delta} \rightarrow 0$ in the product topology, then $u_{\delta_{k}} \rightarrow 0$ for each $\mathrm{k}$, and so

$$
r_{\alpha_{n}, n}\left(x^{\delta}\right)=\sum_{k=1}^{n} q_{\alpha_{n}, N_{k}}\left(u_{\delta_{k}}\right) \rightarrow 0
$$

which proves that $x^{\delta} \rightarrow 0$ in the topology $\tau_{N}$. Conversely, suppose that $x^{\delta} \rightarrow 0$ in the topology $\tau_{N}$ and let $0<\alpha<1$ and $\mathrm{k}$ be given. Choose $n>k$ such that $n /(n+1)>\alpha$. Now

$$
q_{\alpha, N_{k}}\left(u_{\delta_{k}}\right) \leq q_{\alpha_{n}, N_{k}}\left(u_{\delta_{k}}\right) \leq r_{\alpha_{n}, n}\left(x^{\delta}\right) \rightarrow 0 .
$$

This clearly proves that $x^{\delta} \rightarrow 0$ in the product topology.

(4) It follows from the fact that a fuzzy seminorm is a fuzzy norm iff the corresponding topology is Hausdorff together with the well known property that the cartesian product of non-empty topological spaces is Hausdorff iff each factor is Hausdorff. 


\section{Tensor products of fuzzy norms}

Let $N_{1}, N_{2}$ be fuzzy seminorms on the vector spaces $E, F$, respectively. Define $N=N_{1} \otimes N_{2}$ on the tensor product $E \otimes F$ by

$$
N(u)=\wedge\left\{\oplus_{k=1}^{n} N_{1}\left(x_{k}\right) \otimes N_{2}\left(y_{k}\right): u=\sum_{k=1}^{n} x_{k} \otimes y_{k}\right\}
$$

where the infimum is taken over the family of all possible representations $u=\sum_{k=1}^{n} x_{k} \otimes y_{k}$ of $u \in E \otimes F$. We claim that $N$ is a fuzzy seminorm on $g=E \otimes F$.

(FN1) If $u=0=0 \otimes 0$, then $N(u) \preceq N_{1}(0) \odot N_{2}(0)=\tilde{0}$ and so $N(u)=\tilde{0}$.

$\left(\right.$ FN3) Let $c \neq 0$. Then $\left[N_{1}(x) \odot N_{2}(y)\right](t)=\sup _{s>0} N_{2}(y, s) \wedge N_{1}\left(t s^{-1}\right)=$ $\sup _{s>0} N_{2}(y, s) \wedge N_{1}\left(c^{-1} x,|c|^{-1} t / s\right)=\left[N_{1}\left(c^{-1} x\right) \odot N_{2}(y)\right]\left(|c|^{-1} t\right)$. Now

$$
N(c u, t)=\sup \left\{\left[\oplus_{k=1}^{n} N_{1}\left(x_{k}\right) \odot N_{2}\left(y_{k}\right)\right](t): u=\sum_{k=1}^{n} x_{k} \oplus y_{k}\right\} .
$$

For $c u=\sum_{k=1}^{n} x_{k} \oplus y_{k}$ and $z_{k}=c^{-1} x_{k}$, we have $u=\sum_{k=1}^{n} z_{k} \oplus y_{k}$. Thus

$\left[\oplus_{k=1}^{n} N_{1}\left(x_{k}\right) \odot N_{2}\left(y_{k}\right](t)=\sup \left\{\wedge N_{1}\left(x_{k}\right) \odot N_{2}\left(y_{k}\right)\right]\left(t_{k}\right): t=\sum_{k=1}^{n} t_{k}\right\}$

$=\sup \left\{\wedge\left[N_{1}\left(z_{k}\right) \odot N_{2}\left(y_{k}\right)\right]\left(|c|^{-1} t_{k}\right)\right.$. Therefore

$N(c u, t)=\sup \left\{\left[\oplus_{k=1}^{n} N_{1}\left(z_{k}\right) \odot N_{2}\left(y_{k}\right)\right]\left(|c|^{-1} t\right): u=\sum z_{k} \oplus y_{k}\right\}$

$=N\left(u,|c|^{-1} t\right)$.

(FN2) We have that $N(u+v, t+s) \succeq N(u, t) \wedge N(y, s)$. In fact, let $N(u, t) \wedge N(y, s)>\alpha$. There are representations $u=\sum_{k=1}^{n} x_{k} \oplus y_{k}$ and $v=\sum_{k=n+1}^{m} x_{k} \oplus y_{k}$ such that $\xi(t) \wedge \eta(s)>\alpha$, where $\xi=\oplus_{k=1}^{n} N_{1}\left(x_{k}\right) \odot N_{2}\left(y_{k}\right), \eta=$ $\sum_{k=n+1}^{n} N_{1}\left(x_{k}\right) \odot N_{2}\left(y_{k}\right)$. Now $\xi \oplus \eta=\sum_{k=1}^{m} N_{1}\left(x_{k}\right) \odot N_{2}\left(y_{k}\right)$ and $\xi \oplus \eta \geq(\xi \oplus \eta)(t+s)>\alpha$. This proves that $N(u+v) \preceq N(u) \oplus N(v)$ and claim follows.

Theorem 6.1 For $0<\alpha<1$ we have that $q_{\alpha, N}(u)=q_{\alpha, N_{1}} \oplus q_{\alpha, N_{2}}$.

Proof . Suppose that $q_{\alpha, N}(u)<t$. Then $N(u, t)>\alpha$. There exists a representation $u=\sum_{k+1}^{n} x_{k} \oplus y_{k}$ such that

$$
\left[\oplus_{k=1}^{n} N_{1}\left(x_{k}\right) \odot N_{2}\left(y_{k}\right)\right](t)>\alpha .
$$

Now there are $t_{k}, \sum_{k=1}^{n} t_{k}=t$ such that

$$
\bigwedge_{k=1}^{n}\left[N_{1}\left(x_{k}\right) \odot N_{2}\left(y_{k}\right)\right](t)>\alpha
$$

For each $\mathrm{k}$, there exists $s_{k}$ with $\left.N_{1}\left(x_{k}, s_{k}\right) \wedge N_{2}\left(y_{k}\right), t_{k} / s_{k}\right)>\alpha$. Now

$$
q_{\alpha, N_{1}}\left(x_{k}\right)<s_{k}, \quad q_{\alpha, N_{2}}\left(y_{k}\right)<t_{k} / s_{k}
$$

and therefore $q_{\alpha, N_{1}}\left(x_{k}\right) q_{\alpha, N_{2}}\left(y_{k}\right)<t_{k}$, which implies that

$$
q_{\alpha, N_{1}} \oplus q_{\alpha, N_{2}}(u) \leq \sum_{k=1}^{n} q_{\alpha, N_{1}}\left(x_{k}\right) q_{\alpha, N_{2}}\left(y_{k}\right)<\sum_{k=1}^{n} t_{k}=t .
$$

Conversely, suppose $q_{\alpha, N_{1}} \oplus q_{\alpha, N_{2}}(u)<t$. There exists a representation $u=\sum_{k=1}^{n} x_{k} \oplus y_{k}$ such that

$$
\sum_{k=1}^{n} q_{\alpha, N_{1}}\left(x_{k}\right) q_{\alpha, N_{2}}\left(y_{k}\right)<t
$$

Now, there is $\epsilon>0$ such that, for $t_{k}=\epsilon+q_{\alpha, N_{1}}\left(x_{k}\right), s_{k}=\epsilon+q_{\alpha, N_{2}}\left(y_{k}\right)$, we $\sum_{k=1}^{n} t_{k} s_{k}<t$. Then $N_{1}\left(x_{k}, t_{k}\right)>\alpha$, $N_{2}\left(y_{k}, s_{k}\right)>\alpha$, and so $\left[\oplus_{k=1}^{n} N_{1}\left(x_{k}\right) \odot N_{2}\left(y_{k}\right)\right](t) \geq\left[\oplus_{k=1}^{n} N_{1}\left(x_{k}\right) \odot N_{2}\left(y_{k}\right)\left(\sum_{k=1}^{n} t_{k} s_{k}\right)\right.$

$\geq \bigwedge_{k=1}^{n}\left[N_{1}\left(x_{k}\right) \odot N_{2}\left(y_{k}\right)\right]\left(t_{k} s_{k}\right) \geq \bigwedge_{k=1}^{n} N_{1}\left(x_{k}, t_{k}\right) N_{2}\left(y_{k}, s_{k}\right)>\alpha$. The result clearly follows.

Corollary 6.2 For all $x \in E, y \in F$ we have

$$
q_{\alpha, N}(x \otimes y)=q_{\alpha, N_{1}} \otimes q_{\alpha, N_{2}}(x \otimes y)=q_{\alpha, N_{1}}(x) q_{\alpha, N_{2}}(y) .
$$


In view the theorem., $\tau_{N}$ coincides with the projective tensor product topology.

Theorem 6.3 $N(x \otimes y)=N_{1}(x) \odot N_{2}(y)$.

Proof . By the definition of $N$, we have that $N(x \otimes y) \preceq N_{1}(x) \otimes N_{2}(y)$. On the other hand, suppose that $N(x \otimes y, t)>\alpha$. Then $q_{\alpha, N}(x \otimes y)=q_{\alpha, N_{1}}(x) q_{\alpha, N_{2}}(y)<t$. There are $t_{1}>q_{\alpha, N_{1}}(x), t_{2}>q_{\alpha, N_{2}}(y)$ such that $t_{1} t_{2}<t$. Then $N_{1}\left(x, t_{1}\right)>\alpha$ and $N_{2}\left(y, t_{2}\right)>\alpha$, and so $N_{1}(x) \odot N_{2}(y)(t)>\alpha$. This clearly completes the proof.

Theorem 6.4 $N_{1} \oplus N_{2}=N$ is the biggest of all fuzzy seminorms $N^{\prime}$ such that $N^{\prime}(x \times y) \preceq N_{1}(x) \odot N_{2}(y)$ for all $x \in E, y \in F$.

Proof. Suppose that $N^{\prime}(x \otimes y) \preceq N^{\prime}(x) \odot N^{\prime}(y)$ for all $(x, y)$. If $u=\sum_{k=1}^{n} x_{k} \otimes y_{k}$, then

$$
N^{\prime}(u) \preceq \bigoplus_{k=}^{n} N^{\prime}\left(x_{k} \otimes y_{k}\right) \preceq \bigoplus_{k=1}^{n} N_{1}\left(x_{k}\right) \odot N_{2}\left(y_{k}\right) .
$$

It follows from this that $N^{\prime}(u) \preceq N(u)$ for all $u$, as it was to be proved.

\section{References}

[1] Moustafa A. Amer and Nehad N. Morsi, Bounded linear transformations between probabilistic normed linear spaces, Fuzzy Sets and Systems 73(1995, 167-183. bibitem T Bag and S. K. Samanta, Finite dimensional fuzzy normed linear spaces, J. Fuzzy Mathematics 11(3)(2003), 687-205.

[2] T Bag and S. K. Samanta, Fuzzy bounded linear operators, Fuzzy Sets and Systems 151(2005), 513-547.

[3] T Bag and S. K. Samanta,Fixed point theorems in fuzzy normed linear spaces, Inform. Sci. 176(2006), $2910-2931$.

[4] T Bag and S. K. Samanta, A comparative study of fuzzy norms on a linear space, Fuzzy Sets andSystems 159(2008), 670-684.

[5] T Bag and S. K. Samanta, Produt fuzzy normed linear spaces, J. Fuzzy Mathematics 13(3)(2005), 545-565.

[6] T Bag and S. K. Samanta, Some fixed point theorems in fuzzy normed linear spaces, Inform. Sci. 111(2007), 3271-3289.

[7] S. C. Cheng and J. N. Mordeson, Fuizzy linear operators and fuzzy normed linear spaces, Bull. Calc. Math. Soc. 86(1994), 429-436.

[8] C. Felbin, Finite dimensional normed linear spaces, Fuzzy Sets and Sytems 48(1992), 239-248.

[9] C. Felbin, Fuzzy dimensional normed linear spaces II, J. Analysis 7(1999), 117-131.

[10] O. Kaleva and S. Seikkala, On fuzzy metric spaces, Fuzzy Sets and Systems 12(1984), 143-154.

[11] A. K. Katsaras, Fuzzy topological vector spaces II, Fuzzy Sets and Systems 12(1984), 143-154.

[12] Dorel Mihet, The stability of the Cauchyfunctional equation in non-Archimedean fuzzy normed spaces, Fuzzy Sets and Systems 161(2010), 2206-2212.

[13] Alireza Kamel Mirmostafaee and Mohammad Sal Moslehian, Stability of additive mappings in non-Archimedean fuzzy normed spaces, Fuzzy Sets and Systems 160(2009), 1643-1652.

[14] C. Park, Fuzzy stability of a functional equation associated with inner product spaces, Fuzzy Sets and systems 160(2009), 1632-1642.

[15] B. Schweizer and A. Sklar, Probabilistic metric spaces, North Holland, New York, 1983.

[16] J.Xiao and X. Zhu, On linearl;y topological structure and property of fuzzy normed linear spaces, Fuzzy Sets and Systems 125(2002), 153-161.

[17] J. Xiao and X. Zhu, Fuzzy normed space of operators and its completeness, Fuzzy Sets and Systemas 133(2003), 389-399. 